\title{
Adhesive transitions in Newton black films: A computer simulation study
}

\author{
Fernando Bresme, ${ }^{1, a)}$ Enrique Chacón, ${ }^{2, b)}$ Héctor Martínez, ${ }^{3, c}$ and Pedro Tarazona ${ }^{4, d)}$ \\ ${ }^{1}$ Department of Chemistry, Imperial College London, SW7 2AZ, London, United Kingdom \\ ${ }^{2}$ Instituto de Ciencia de Materiales de Madrid, CSIC, 28049, Madrid, Spain and Instituto de Ciencia \\ de Materiales Nicolaś Cabrera, Universidad Autońoma de Madrid, Madrid, 28049, Spain \\ ${ }^{3}$ Departamento de Física Teórica de la Materia Condensada, Universidad Autońoma de Madrid, Madrid, \\ 28049, Spain \\ ${ }^{4}$ Departamento de Física Teórica de la Materia Condensada, Universidad Autońoma de Madrid, Madrid, \\ 28049, Spain and Instituto de Ciencia de Materiales Nicolaś Cabrera, Universidad Autońoma de Madrid, \\ Madrid, 28049, Spain
}

(Received 4 April 2011; accepted 13 May 2011; published online 6 June 2011)

\begin{abstract}
We report molecular dynamics simulations of Newton black films (NBFs), ultra thin films of aqueous solutions stabilized with two monolayers of ionic surfactants, sodium dodecyl sulfate. We show that at low water content conditions and areas per surfactant corresponding to experimental estimates in NBFs, homogeneous films undergo an adhesion "transition," which results in a very thin adhesive film coexisting with a thicker film. We identify the adhesive film with the equilibrium structure of the Newton black film. We provide here a direct microscopic view of the formation of these important structures, which have been observed in experimental studies of emulsions and foams. We also report a detailed investigation of the structural properties and interfacial fluctuation spectrum of the adhesive film. Our analysis relies on the definition of an "intrinsic surface," which is used to remove the averaging effect that the capillary waves have on the film properties. () 2011 American Institute of Physics. [doi:10.1063/1.3596752]
\end{abstract}

\section{INTRODUCTION}

Blacks films (BFs) can form spontaneously from soap solutions. Often they consist of a thin aqueous core, sandwiched between two monolayers of highly packed ionic surfactants, e.g., sodium dodecyl sulfate (SDS), ${ }^{1}$ although there is evidence that BFs can also form with other surfactants ${ }^{2-4}$ as well as phospholipids, ${ }^{5}$ showing the relevance of these structures in both materials science and biology. At low temperatures and high salt concentrations, BFs can undergo transitions to thinner films, typically $\lesssim 40 \AA$ thick. These structures are called Newton black films (NBFs). Experiments have shown that NBFs can be fairly stable in the absence of water evaporation and mechanical disturbances. Understanding their properties is of practical relevance to predicting the stability of a wide range of materials used in food stuffs, oil recovery, rheology, pharmaceutical formulations, blood substitutes, agrochemical industry, or paints.

Many studies have tried to quantify the surface forces operating in black films. The dependence of the common black film thickness with salt concentration is well described by the Poisson-Boltzmann theory. ${ }^{3,6-8}$ Significant departures from this theory have been found in NBFs though, ${ }^{9}$ indicating a breakdown of the double layer picture in these thin films. Computer simulations of ionic NBFs have also shown that water is strongly polarized inside the films, showing that water cannot be modeled as a dielectric continuum with a pre-defined dielectric constant. ${ }^{10,11}$ Hence, models such as

a)Electronic mail: f.bresme@ imperial.ac.uk.

b)Electronic mail: echacon@icmm.csic.es.

c)Electronic mail: hector.martinez@uam.es.

d) Electronic mail: pedro.tarazona@uam.es. the DLVO theory, which includes both double layer interactions and a dielectric continuum, should and indeed fail in the description of the repulsive interactions measured in NBFs experiments. ${ }^{9,12}$ This conclusion should apply to other confined systems, e.g., DNA columnar phases, ${ }^{13}$ which show similar repulsive forces when the distance between the DNA molecules is $<10 \AA$. To explain the repulsion a new type of force, hydration forces, has been introduced. It was argued that the physical origin of the force is connected to the perturbation of the orientation of the water molecules when they are confined in small volumes. ${ }^{11,14,15}$ Alternative explanations based on steric interactions have also been proposed (see, e.g., Refs. 1 and 16).

In this work we investigate using computer simulations the structure of sodium dodecyl sulfate Newton black films (SDS-NBFs) as a function of the film water content. This system is of great interest to us, as experimental studies have shown that SDS surfactants form Newton black films in both foams ${ }^{17}$ and emulsions. ${ }^{18}$ Moreover the structural properties of the films as well as their adhesive behavior (contact angles and adhesive energy) are similar. ${ }^{19,20}$ This observation indicates that the nature of the phases in contact and their different Hamaker constants (air in foams vs. oil in emulsions) does not play a relevant role in determining the adhesion strength. Instead, the monolayer-monolayer interactions would determine the physical behavior of these bilayers at short interlayer distances. This is an interesting notion that has provided a principle to modify the stability of foams/emulsions, by specifically targeting the surfactant properties.

Computer simulations provide a powerful approach to investigate the structure and stability of NBFs. Most computer simulations of SDS-NBFs have focused so far on the 
investigation of the structure of foam films..$^{10,11,21-26}$ The simulated structures, with thicknesses similar to those inferred from $x$-ray experimental data, ${ }^{17}$ indicate that the film contains between two and four water molecules per surfactant. Part of this water accumulates around the head groups as solvation water, the remaining distributing in the film's core. All the simulations referred to above have investigated the structure of the films by computing the "mean" density profiles. Because the films are soft and undergo thermal fluctuations (capillary waves) the mean profiles can only provide an average view of the water structure next to the surfactant layers. A more precise characterization of the "real" structure of water and ions inside the film is desirable though, as this can provide a reference to test current theoretical models of thin films. We have overcome the limitations associated with the computation of mean density profiles, by computing the intrinsic density profile. We employ a computational approach that removes the thermal averaging associated with the capillary waves. One added advantage of this method is the possibility of quantifying the mean square fluctuations of the black films, and from the analysis of these fluctuations one can quantify the film bending modulus. The bending modulus is a relevant quantity in the description of steric forces, which influence the stability of thin films, ${ }^{1}$ as well as in free energy approaches used to explain hole formation in these films. ${ }^{27}$

In this article we investigate using classical molecular dynamics computer simulations the structure, elasticity, and adhesive behavior of SDS-NBFs. We show that NBFs, with surfactant surface concentrations similar to those found in experiments (33 $\AA^{2}$ per surfactant), ${ }^{17}$ undergo adhesion transitions. A full discussion of the thermodynamics of this adhesion"transition" and the corresponding formation of equilibrium states will be reported in a forthcoming article.

\section{METHODOLOGY}

\section{A. Force-field and simulation details}

Our NBFs consist of a thin aqueous core confined between two layers of ionic surfactants, sodium dodecyl sulfate. The force-field used to model this black film has been extensively discussed in previous articles. ${ }^{11,22,23,28}$ We model the surfactants using a united atom approach, where the $\mathrm{CH}_{3}$ and $\mathrm{CH}_{2}$ groups are represented by pseudoatoms. The aliphatic chain is modeled using a combination of bond constraints and harmonic bending potentials, as well as a dihedral potential. We used the TraPPE force-field to model the alkyl chain intramolecular contributions. ${ }^{29}$ The surfactant head group is modeled using parameters from the Assisted Model Building with Energy Refinement (AMBER) forcefield, ${ }^{30}$ including partial charges computed by Shelley et al. using quantum mechanical computations. ${ }^{31}$ The $\mathrm{Na}^{+}$counterion is modeled using the parameters reported by Dang. ${ }^{32}$ A summary of all the parameters employed to model the surfactant are given in Tables I and II. For water we have employed the recently introduced TIP4P-2005 model, ${ }^{33}$ which performs better than its predecessors in predicting the water surface tension. Otherwise, the interfacial structure of TIP4P-2005 is comparable to that obtained with other popular models such as the SPC/E
TABLE I. Simulation parameters used to model the intermolecular interactions of the SDS surfactants.

\begin{tabular}{lllcc}
\hline \hline (Pseudo)Atom & $\sigma(\AA)$ & $\epsilon(\mathrm{kJ} / \mathrm{mol})$ & $\mathrm{q} / \mathrm{e}$ & Reference \\
\hline $\mathrm{CH}_{3}$ & 3.75 & 0.8149 & 0 & 29 \\
$\mathrm{CH}_{2}$ & 3.95 & 0.3825 & 0 & 29 \\
$\mathrm{CH}_{2}$ bonded to O(ester) & 3.95 & 0.3825 & 0.137 & 29,31 \\
$\mathrm{O}($ ester$)$ & 3.3224 & 0.7133 & -0.459 & 30,31 \\
$\mathrm{~S}$ & 4 & 1.046 & 1.284 & 30,31 \\
$\mathrm{O}\left(\mathrm{SO}_{4}^{-}\right)$ & 3.3224 & 0.8786 & -0.654 & 30,31 \\
$\mathrm{Na}^{+}$ & 2.584 & 0.4179 & 1 & 32 \\
\hline \hline
\end{tabular}

(see, e.g., Ref. 34 for a comparison of the intrinsic structures of these two models).

The intermolecular interactions were modeled using a combination of Lennard-Jones and coulombic potentials. We have employed the truncated and shifted Lennard-Jones potential to compute the dispersion interactions,

$$
\begin{gathered}
U_{i j}(r)=4 \varepsilon_{i j}\left[\left(\frac{\sigma_{i j}}{r}\right)^{12}-\left(\frac{\sigma_{i j}}{r}\right)^{6}\right] \text { for } r \leq r_{c}, \\
U_{i j}(r)=0 \text { for } r>r_{c},
\end{gathered}
$$

where $\sigma_{i j}$ and $\varepsilon_{i j}$ represent the effective diameter and interaction strength between atoms of species $i$ and $j$, and $r_{c}$ is the cut-off. The interactions between atoms of different species were derived using standard combining rules, $\sigma_{i j}=\sigma_{i}+\sigma_{j}$, and $\epsilon_{i j}=\sqrt{\epsilon_{i} \epsilon_{j}}$. The short range interactions were cut-off at $14 \AA$, and the electrostatic interactions were computed using the particle mesh Ewald method. ${ }^{35}$ Our simulations involved large systems, consisting of $\mathrm{N}_{s}=512$ surfactants, 256 per monolayer, the corresponding sodium counterions, and $\mathrm{N}_{w}$ water molecules. We performed simulations with larger systems, up to 2048 surfactants, to test the reproducibility of the adhesive states. The amount of water in the film was varied from $\mathrm{n}_{w}=\mathrm{N}_{w} / \mathrm{N}_{s}=0$ to 11.96 water molecules per surfactant, in order to investigate the dependence of the structure and mechanical properties of the NBF on water content. The surfactant surface concentration was set to $33 \AA^{2}$, which corresponds to the area observed in the spontaneous formation of the sodium dodecyl sulfate Newton black films. ${ }^{17}$ All the simulations were performed at $298 \mathrm{~K}$. Long equilibration times ( $>10 \mathrm{~ns}$ ) were considered before computing average properties over at least $10 \mathrm{~ns}$ trajectories. The equations of motion were integrated using the leap-frog algorithm, with a time step of $2 \mathrm{~s}$.

In the rest of the article we will use $\sigma=3.116 \AA$ (which corresponds approximately to the water molecule diameter) to represent the reduced intrinsic density profiles, $\tilde{\rho}^{*}=\rho \sigma^{3}$ and the reduced distances $z / \sigma$. The simulations were performed in parallel using GROMACS $4.5 .^{36}$

\section{B. Intrinsic profiles and estimation of the aqueous core film volume}

The computations of the intrinsic density profile require the construction of the intrinsic surface (IS), $\xi(\mathbf{R})$, where $\mathbf{R}=(x, y)$ represents the location of the surface for a specific 
TABLE II. Simulation parameters used to model the intramolecular interactions of the SDS surfactants. Angular terms are modeled using a harmonic potential $U(\theta)=k_{\theta}\left(\theta-\theta_{e}\right)^{2}$ and dihedral terms using two sets of potentials: $U(\phi)=k_{1} / 2(1+\cos \phi)+k_{2} / 2(1-\cos 2 \phi)+k_{3} / 2(1+\cos 3 \phi)$ and $U(\phi)=k_{4}(1+\cos 3 \phi)$.

\begin{tabular}{|c|c|c|c|c|}
\hline Bond & $r_{e}(\AA)$ & & & Reference \\
\hline$\overline{\mathrm{CH}_{3}-\mathrm{CH}_{2}}$ & 1.54 & & & 29 \\
\hline $\mathrm{CH}_{2}-\mathrm{CH}_{2}$ & 1.54 & & & 29 \\
\hline $\mathrm{CH}_{2}-\mathrm{O}$ (ester) & 1.41 & & & 30 \\
\hline $\mathrm{O}($ ester)-S & 1.58 & & & 30 \\
\hline $\mathrm{S}-\mathrm{O}\left(\mathrm{SO}_{4}^{-}\right)$ & 1.46 & & & 53 \\
\hline Angle & $k_{\theta}\left(\mathrm{kJ} / \mathrm{mol} \mathrm{deg}^{-2}\right)$ & $\theta_{e}(\mathrm{deg})$ & & Reference \\
\hline$\overline{\mathrm{CH}_{3}-\mathrm{CH}_{2}-\mathrm{CH}_{2}}$ & 259.86 & 114 & & 29 \\
\hline $\mathrm{CH}_{2}-\mathrm{CH}_{2}-\mathrm{CH}_{2}$ & 259.86 & 114 & & 29 \\
\hline $\mathrm{CH}_{2}-\mathrm{CH}_{2}-\mathrm{O}($ ester $)$ & 209.2 & 109.5 & & 30 \\
\hline $\mathrm{CH}_{2}-\mathrm{O}($ ester $)-\mathrm{S}$ & 209.2 & 112.6 & & 30 \\
\hline $\mathrm{O}($ ester$)-\mathrm{S}-\mathrm{O}\left(\mathrm{SO}_{4}^{-}\right)$ & 213.5 & 102.6 & & 30 \\
\hline $\mathrm{O}\left(\mathrm{SO}_{4}^{-}\right)-\mathrm{S}-\mathrm{O}\left(\mathrm{SO}_{4}^{-}\right)$ & 213.5 & 115.4 & & 30 \\
\hline Torsion & $k_{1}\left(\mathrm{~kJ} / \mathrm{mol} \mathrm{deg}^{-2}\right)$ & $k_{1}\left(\mathrm{~kJ} / \mathrm{mol} \mathrm{deg}^{-2}\right)$ & $k_{1}\left(\mathrm{~kJ} / \mathrm{mol} \mathrm{deg}^{-2}\right)$ & Reference \\
\hline $\mathrm{CH}_{3}-\mathrm{CH}_{2}-\mathrm{CH}_{2}-\mathrm{CH}_{2}$ & 5.905 & -1.134 & 13.161 & 29 \\
\hline $\mathrm{CH}_{2}-\mathrm{CH}_{2}-\mathrm{CH}_{2}-\mathrm{CH}_{2}$ & 5.905 & -1.134 & 13.161 & 29 \\
\hline Torsion & $k_{4}\left(\mathrm{~kJ} / \mathrm{mol} \mathrm{deg}^{-2}\right)$ & & & Reference \\
\hline 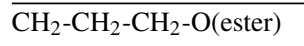 & 11.7152 & & & 53 \\
\hline $\mathrm{CH}_{2}-\mathrm{CH}_{2}-\mathrm{O}$ (ester)-S & 9.6232 & & & 53 \\
\hline $\mathrm{CH}_{2}-\mathrm{O}$ (ester)-S-O $\left(\mathrm{SO}_{4}^{-}\right)$ & 2.092 & & & 53 \\
\hline
\end{tabular}

configuration. By referring the particle coordinates to the intrinsic surface, it is possible to construct the intrinsic density profile, ${ }^{37}$

$$
\tilde{\rho}(z)=\left\langle\frac{1}{A_{0}} \sum_{i=1}^{N} \delta\left(z-z_{i}+\xi\left(\mathbf{R}_{i}\right)\right)\right\rangle,
$$

where $A_{0}$ is the cross sectional area of the interface. The intrinsic density profile is not affected by the blurring effect of the capillary wave fluctuations, and therefore provides a much better resolution of the interfacial structure. We note that computer simulations of ionic liquids and molecular fluid interfaces ${ }^{38-40}$ have shown that the interfacial fluctuations of the simulated interfaces are fully consistent with the capillary wave theory predictions (see, e.g., Ref. 41).

Different approaches have been developed to identify the IS. ${ }^{37,42-48}$ The construction of the IS in the case of Newton black films is simpler, as we can use the surfactant head (in our case the sulfur atoms) to define the IS for each monolayer. ${ }^{28}$ This represents a natural choice as the surfactants never leave the interface during the simulation. In this work we construct the IS using a three-dimensional triangulation method. The procedure has been discussed in Ref. 49 and in Ref. 28 for the specific case of SDS monolayers. We compute first a two-dimensional Delaunay triangulation using the coordinates of the sulfur atoms projected on the interface plane. The Delaunay triangulation is used to identify the nearest neighbors of a given atom and to construct a triangulated surface, where the triangle edges join a given sulfur atom to its nearest neighbors. Applying the same procedure to the surfactant heads on the two layers of the film, we get the two intrinsic surfaces $\xi^{a}(\mathbf{R})$ and $\xi^{b}(\mathbf{R})$. The mean normal distance between these two surfaces, $\langle h\rangle=\left\langle\xi^{a}(\mathbf{R})-\xi^{b}(\mathbf{R})\right\rangle$, provides a route to estimate the volume of the aqueous layer confined inside the film $A_{0}\langle h\rangle$.

\section{RESULTS}

\section{A. Intrinsic structure of NBF}

Figure 1 represents the intrinsic density profiles of Newton black films as a function of the water content. As discussed in Sec. II B the intrinsic profiles are computed using the intrinsic surface as a reference surface. We represent the intrinsic profiles with respect to one of these surfaces, hence the profiles are asymmetric. At short distance the intrinsic structure is clearly observed, whereas at long distances when correlations with the intrinsic surface disappear, the profiles resemble the traditional mean profile.

The intrinsic profile features two well defined water layers around the surfactant head group $(\mathrm{z} \sim 0)$. The density profile in the aqueous region resembles the profile of water next to rigid surfaces, featuring regular oscillations, which decay to the bulk density in about $3 \sigma$ units $(\sim 10 \AA$ ) (see system $\left.\mathrm{n}_{w}=11.96\right)$. We find that the water structure of the largest thin film investigated here $\mathrm{n}_{w}=11.96$, fully agrees with that found in SDS monolayers at the same area per surfactant. ${ }^{28}$ Confinement effects do not have a major influence on the water solvation structure of thinner films either (down to $\left.\mathrm{n}_{w}=8.6\right)$. For lower water contents $\left(\mathrm{n}_{w} \leq 6\right)$ the structure of the aqueous core $((z>0)$ is severely disrupted, but interestingly the solvation layer in the aliphatic region $(\mathrm{z}<0)$ is not affected, even for very low water contents, $\mathrm{n}_{w}=2$.

To advance in the description of the film structure we have computed the intrinsic orientational profiles,

$$
\tilde{P}(z)=\left\langle\sum_{i=1}^{N} \frac{\hat{\mathbf{p}}_{i} \hat{\mathbf{z}}}{A_{0}} \delta\left(z-z_{i}+\xi\left(\mathbf{R}_{i}\right)\right)\right\rangle,
$$



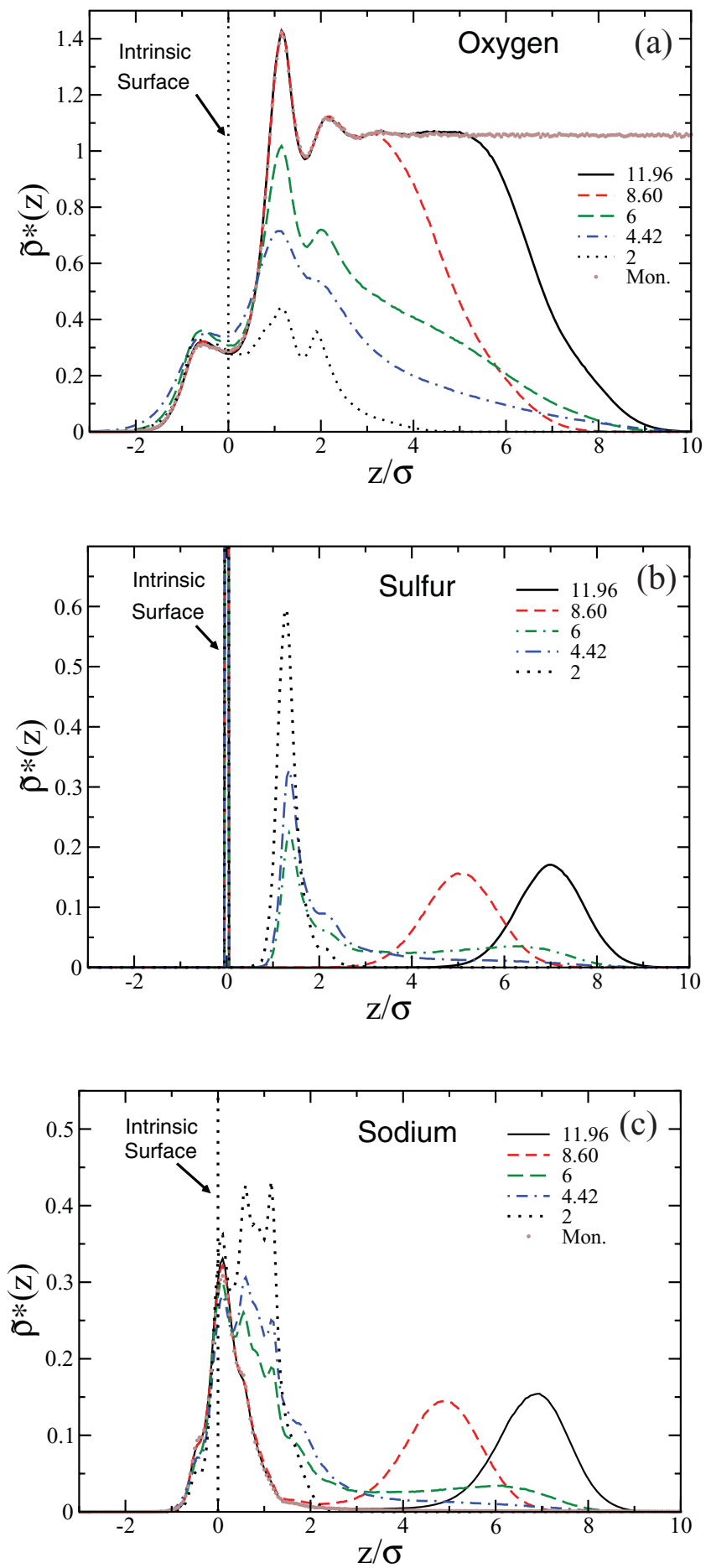

FIG. 1. Intrinsic structure of Newton black films as a function of water content, $\mathrm{n}_{w}=11.96,8.6,6,4.42$, and 2 . The intrinsic density profile of the SDS monolayer at the same area per surfactant is also shown (Ref. 28). From top to bottom: oxygen intrinsic density profiles, sulfur and sodium intrinsic density profiles.

where $\hat{\mathbf{p}}_{i}$ is defined in water as the "unit" vector pointing in the direction of the dipole moment and $\hat{\mathbf{z}}$ is the unit vector normal to the film plane. The second moment distribution profile is given by

$$
\tilde{T}(z)=\left\langle\sum_{i=1}^{N} \frac{3\left(\hat{\mathbf{p}}_{i} \hat{\mathbf{z}}\right)^{2}-1}{2 A_{0}} \delta\left(z-z_{i}+\xi\left(\mathbf{R}_{i}\right)\right)\right\rangle,
$$

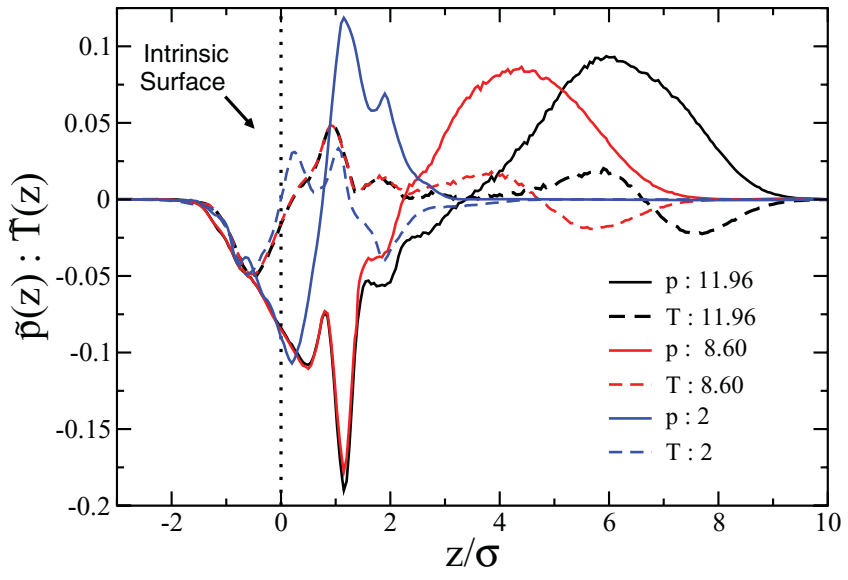

FIG. 2. Water intrinsic orientational profiles as a function of water content $\mathrm{n}_{w}=11.96,8.6,6,4.42$, and 2 .

which indicates whether the water molecules are oriented perpendicular $(\tilde{T}>0)$ or planar $(\tilde{T}<0)$ to the interface. We note that the profiles calculated through Eqs. (4) and (5) are weighted by the density, as this computation provides a more direct comparison with experimental studies.

The orientational profiles show that the water molecules solvating the head groups exhibit a strong orientational ordering (see Figure 2). The dipoles in both the aqueous and aliphatic phase regions $(-1<z / \sigma<1)$ point preferentially towards the aliphatic region of the film. The polarization reaches a maximum close to the SDS head groups and propagates inside the aqueous core. Given the symmetry of the black film, the water molecules reverse their orientation in the middle of the film, where the dipole moment is zero. This general behavior is reproduced in a wide range of water content conditions. Interestingly the orientation in the aliphatic region is preserved for very low water content conditions $\left(\mathrm{n}_{w}=2\right)$. The second moment, $\tilde{T}$ offers complementary information on the water solvation layer. The plane of the water molecules located in the aliphatic region is parallel to the NBF plane, whereas it is perpendicular to this plane in the aqueous region. In agreement with previous observations ${ }^{10}$ we find that the water molecules show a preferred orientation everywhere in the film, "even" in regions where the density reaches bulk values.

\section{B. Evidence for adhesive behavior in Newton black films}

Further analysis of the intrinsic profiles with water content $\mathrm{n}_{w} \leq 6$ shows that the oxygen density decays very slowly for $z>3 \sigma$ (see Figure 1). This behavior might be connected to the presence of regions of different thickness inside the film. This idea is confirmed by inspecting the sulfur intrinsic profiles (see Figure 1), which show the density of one monolayer referred to the intrinsic surface defined by the other monolayer. At high water content $\left(\mathrm{n}_{w} \geq 8.6\right)$ the sulfur atom profile is defined by a Gaussian distribution, and the location of the two monolayers is well defined. The Gaussian behavior agrees with that observed in surfactant monolayers, ${ }^{28}$ and 

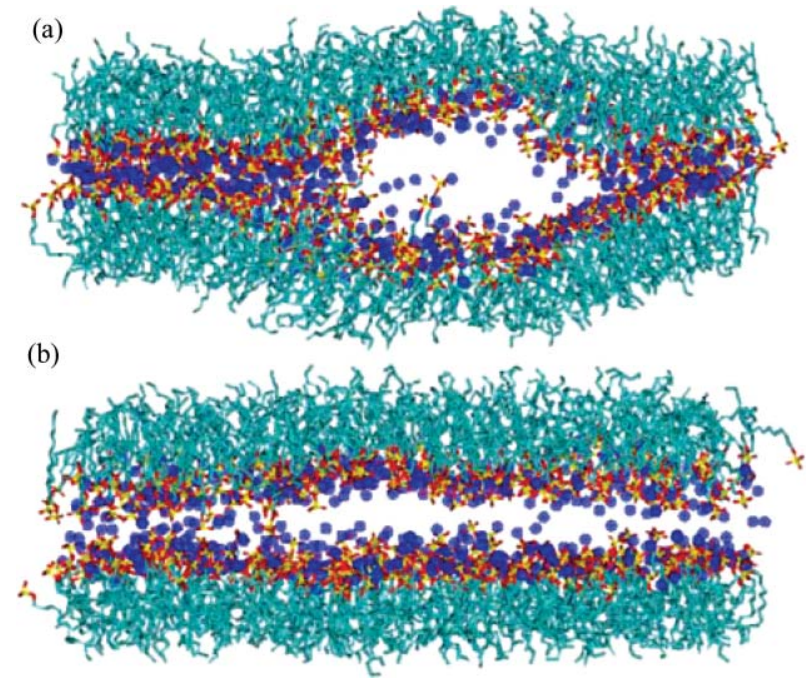

FIG. 3. Snapshots of Newton black films with $\mathrm{n}_{w}=6$. After $24 \mathrm{~ns}$ (top) and 1 ns (bottom).

indicates that the fluctuations of the two monolayers are uncorrelated. We will quantify this point in Sec. III E.

For smaller water content, $\mathrm{n}_{w}=6$, the sulfur density profile is very different. There is a main peak next to the intrinsic surface, showing that the two surfaces are almost in "contact," but we also find a slow decay in the sulfur density at longer distances $z>3 \sigma$ followed by a second maximum. Similar trends are observed in the sodium intrinsic profile (see Figure 1). These results indicate that the film has developed two regions of different thicknesses. This idea is illustrated in Figure 3, which shows the structural changes undergone by the $\mathrm{n}_{w}=6$ film at two different stages, after $1 \mathrm{~ns}$ and $24 \mathrm{~ns}$. We find that whenever the water content is lower than $\mathrm{n}_{w} \approx$ 6 the film becomes unstable and the two monolayers stick together. The water molecules between these adhesive layers are expelled forming a cylindrical or spherical lens inside the film. The structural changes observed in the intrinsic profiles along with the snapshots clearly indicate the existence of $a d$ hesion in the SDS-NBF. Such adhesive behavior is compatible with investigations of oil-water emulsions stabilized with SDS surfactants, ${ }^{18}$ and to our knowledge it is the first time it has been reported in computer simulations.

We have characterized the dynamics of the adhesion process by computing a two-dimensional histogram of the distance between the intrinsic surfaces as a function of the position on the film plane. In this way we can follow the temporal evolution of the film thickness in different regions inside the film. Figure 4 (top) shows the plot for the system containing 512 surfactants. Starting from a homogeneous film, we observe changes in the film thickness within $1 \mathrm{~ns}$. The film evolves into a structure with two well defined regions, high and low water content, which corresponds to the water lens observed in Figure 3. The film structure then evolves slowly for the whole duration of the simulation $(11 \mathrm{~ns})$. We note that the final state of the film is determined by the film surface area and the water content. In addition, the final state might be influenced by the simulation box area. To address this finite size effect we have investigated a larger film, consisting of 2048 surfactants, i.e., an area four times larger than the one discussed above. The resulting system contains 85968 water molecules. Figure 4 represents snapshots of the water film in the interval 16-172 ns. From thermodynamic arguments we expect that the water expelled during the adhesion process will form a droplet when the system reaches equilibrium. It is remarkable this process can be followed in the atomistic simulations. Figure 4 (bottom) clearly shows the formation of the droplet, which requires simulation times in the $100 \mathrm{~ns}$ timescale. We thus confirm that the adhesion process is observed in both small and large systems, hence giving further support to the physical significance of our finding.

The adhesion process results in a film with a very heterogeneous structure, featuring large lateral variations in the film thickness (see Figure 4). In order to characterize the local structure of water in the film during the thinning process, we have computed the partial intrinsic density profile $\tilde{\rho}_{h}(z)$ as the intrinsic profile corresponding to points $(x, y)$ on the film plane, where the local distance between the intrinsic surfaces is given by $h-0.1 \sigma<h(x, y)=\mid \xi^{a}(x, y)-$ $\xi^{b}(x, y) \mid<h+0.1 \sigma$. For this computation we have used 4000 configurations of the adhesive film $\left(\mathrm{n}_{w}=6\right)$ containing 512 surfactants and a grid size of $1 \sigma^{2}$. Although the film thickness locally evolves with time, we note that the density profile obtained for specific separations between the intrinsic surfaces, $\tilde{\rho}_{h}(z)$, does not change with the time interval used. This indicates that the profile $\tilde{\rho}_{h}(z)$ for a specific $h$ is a well defined quantity. The local structure for separations, $\left|\xi^{a}(x, y)-\xi^{b}(x, y)\right|=8 \sigma$ agrees with the structure obtained in homogeneous films, i.e., non-adhesive films $\left(\mathrm{n}_{w}=11.92\right.$, see Figures 1 and 5). We also find good agreement at smaller thicknesses, see films $6 \sigma$ and $1.25 \sigma$ in Figure 5. We emphasize that the results obtained in the adhesive film correspond to averages over a very small region. Despite this the local structure agrees with that of an extended homogeneous film with much larger area $\left(92 \times 92 \AA^{2}\right)$. Hence, the local structure is determined mainly by the film thickness, and our results point towards a rapid evolution of the water structure as a response to the variation of the film thickness, or in other words "local equilibrium" during the thinning process. The rapid diffusion of water inside the thicker films ${ }^{22}$ must enable the establishment of this local equilibrium. Again, the intrinsic profiles show that the decrease in water content (see profiles 2-3 $\sigma$ in Figure 5) does not affect the water structure in the aliphatic region $(-2<z / \sigma \lesssim 1)$. Nevertheless for very low water content we find that water accumulates in the aliphatic region forming a double layer. For these states the head groups and the counterions of both surfactant layers are in direct contact.

\section{Solvation structure of the NBF and the adhesive state}

In this section we examine in more detail the solvation structure of the Newton black films. We recall that the structure of water in the aliphatic region (see Figure 1), $z<0$, is independent of water content for a wide range of film thicknesses $\left(\mathrm{n}_{w} \geq 2\right)$. Using the intrinsic density profiles we have calculated the amount of solvation water in that region by 

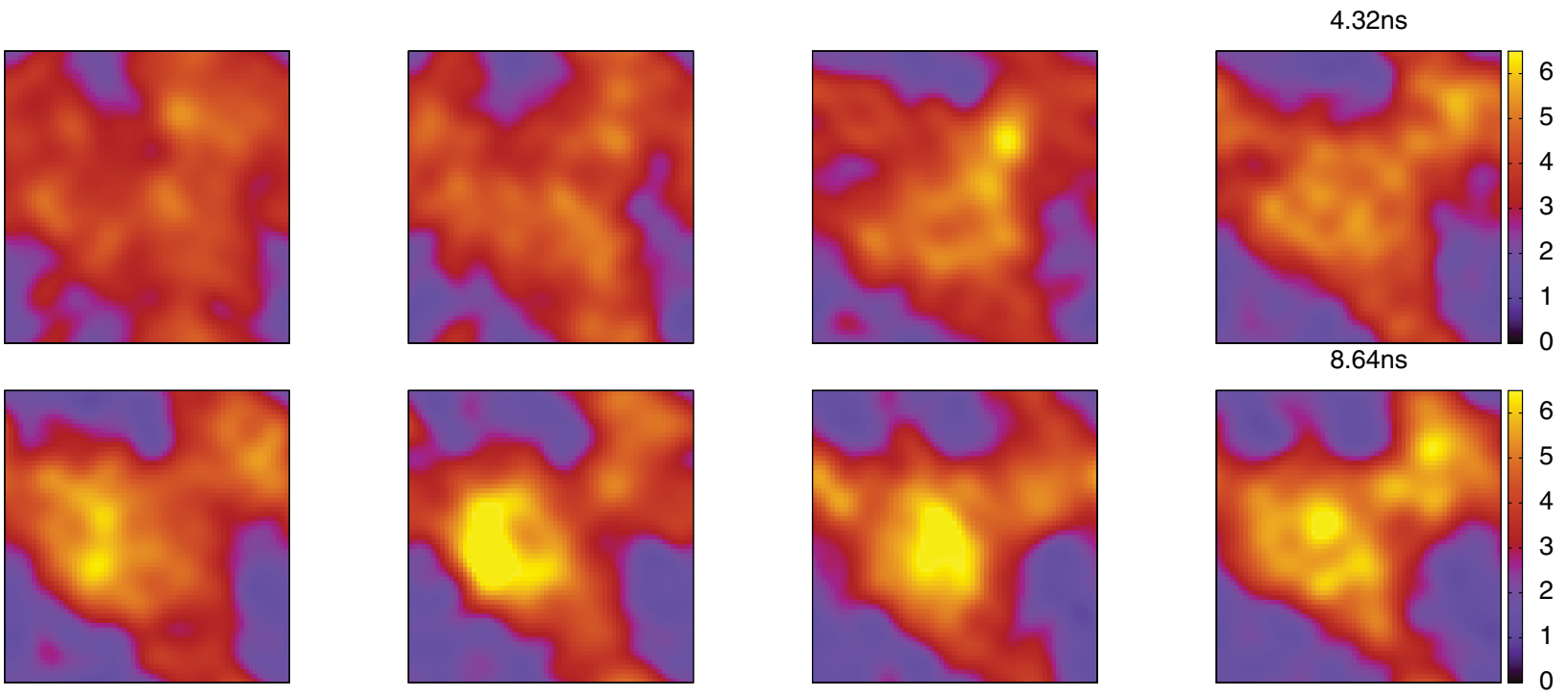

(a)
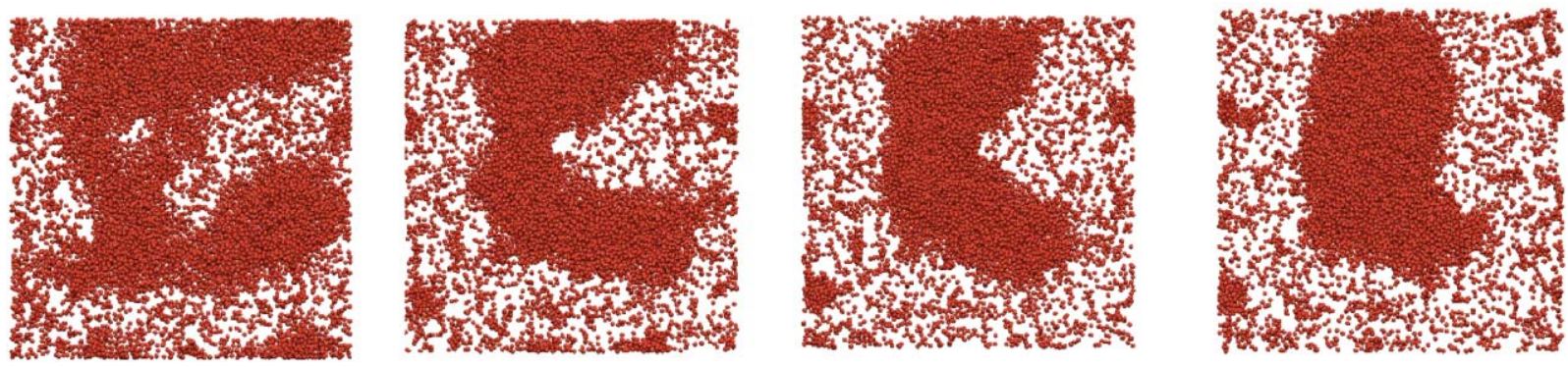

$16 \mathrm{~ns}$
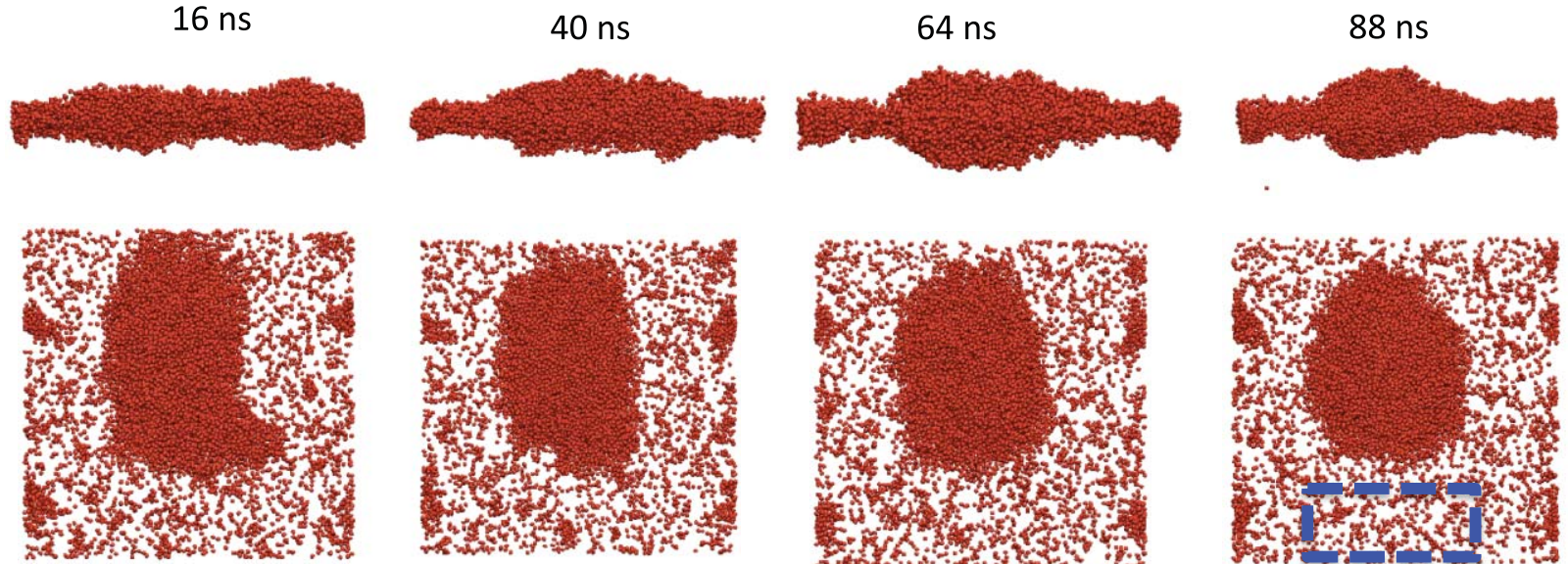

$100 \mathrm{~ns}$

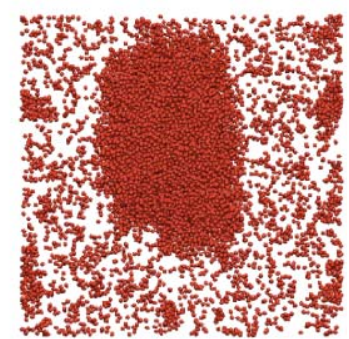

$124 \mathrm{~ns}$
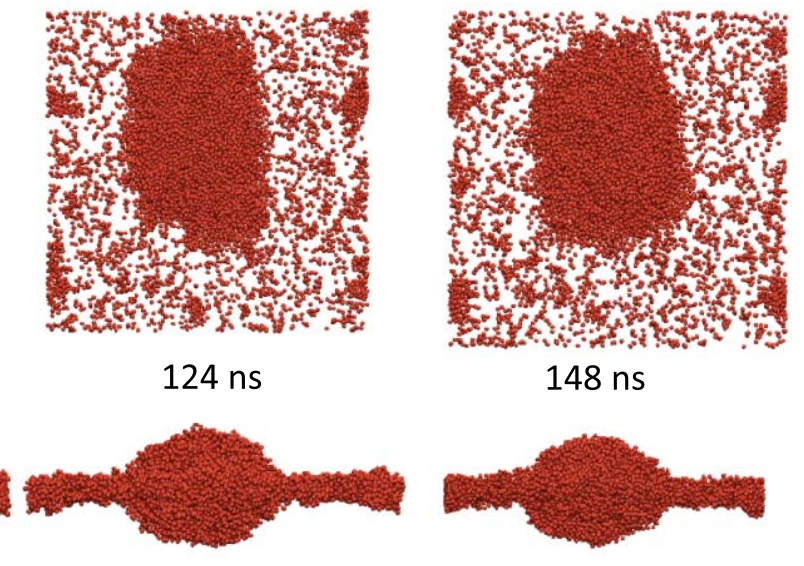

$148 \mathrm{~ns}$

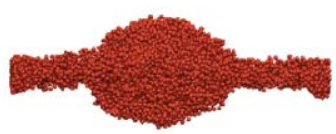

(b)

FIG. 4. (Top): Evolution of the film thickness $h(x, y)$ with time for the small system containing 512 surfactants. Regions of different thicknesses are represented with different colors: yellow, $h(x, y)=6$ and blue, $h(x, y)=0 \sigma$. See right panels for color scale. The grid size used to represent the thickness is $1 \sigma^{2}$. (Bottom): Snapshots showing the time evolution of the film and the formation of the water lens for the large system containing 2048 surfactants. The points represent the oxygen atoms in the water molecule. In both cases the water content corresponds to $\mathrm{n}_{w}=6$ and area per surfactant $33 \AA^{2}$. The rectangle in the lower panel represents the location of the test volume used to estimate the area per surfactant and the number of water molecules per surfactant in the adhesive state. 


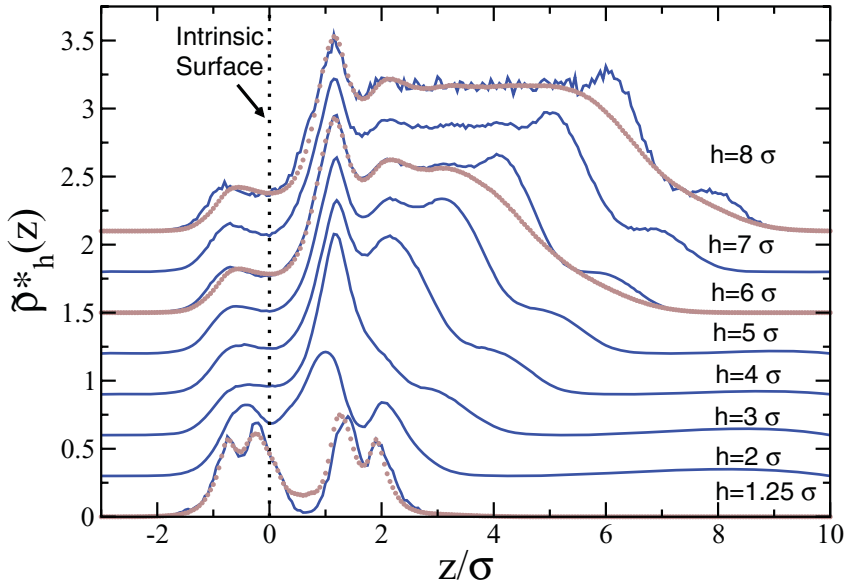

FIG. 5. The lines show the water oxygen partial intrinsic density profiles obtained with the requirement that the local distance between the intrinsic surfaces is $h, \tilde{\rho}_{h}(z)$, from the analysis of the adhesive film, $\mathrm{n}_{w}=6$. The corresponding value of $\mathrm{h}$ is labeled above each curve. The circles represent the intrinsic density profiles $\tilde{\rho}(z)$ obtained from separate simulations of homogeneous films containing 512 surfactants and different amounts of water, $\mathrm{n}_{w}=11.96(\langle h\rangle \approx 8 \sigma), \mathrm{n}_{w}=8.6(\langle h\rangle \approx 6 \sigma)$ and $\mathrm{n}_{w}=2(\langle h\rangle \approx 1.25 \sigma)$.

integrating the density profiles up to the first minimum, $z=0$. We obtain: $1.1,1.1,1.2,1.4$, and 1.2 for $\mathrm{n}_{w}=11.86,8.60$, $6,4.4$, and 2 respectively, which is in good agreement with previous simulation estimates using mean profiles. ${ }^{22}$ It is obvious that the water adsorbed in the aliphatic region or in the plane of the surfactant heads does not contribute to the volume of the film core. Once the monolayers are fully solvated, any water excess will increase the distance between the two monolayer intrinsic surfaces, i.e., the volume of the aqueous core. The top panel in Figure 6 represents the mean separation of the sulfur intrinsic surfaces $\langle h\rangle$ as a function of water content, $n_{w}$. The procedure to compute the mean separation has been discussed in Sec. II B. At high water content the film thickness grows linearly with $n_{w}$, in agreement with previous observations. ${ }^{22}$ At low water content, $\mathrm{n}_{w} \leq 2$ the thickness of the film is very similar to that of the complete dehydrated system, $\mathrm{n}_{w}=0$ and $\langle h\rangle=0.99$. This result indicates that there is an amount of water that does not contribute to the film volume, possibly because it is present in the aliphatic side of the film. This notion is consistent with the results presented in Figure 5, which showed the absence of water between water monolayers when $\mathrm{n}_{w}<2$. We will refer to these water molecules as "hidden" water, $\mathrm{N}_{h}$, to emphasize it does not contribute to the film thickness. The variation of the film thickness with water content can be described using the following equation:

$$
\left\langle h\left(n_{w}\right)\right\rangle=h_{0}+\left(n_{w}-n_{h}\right) /\left(A \rho_{w}\right),
$$

where $\rho_{w}$ is the bulk density of water, $n_{h}=N_{h} / N_{s}$, and $A$ the film surface area per surfactant, $33 \AA^{2}$. The fitting of the simulation data $\mathrm{n}_{w}>3$ to this equation is shown in Figure 6top panel. From the fitting we obtain $\mathrm{n}_{h}=2$. There is a clear deviation of the simulation data from the linear behavior at low water content, $\mathrm{n}_{w}<3$, this indicates that $n_{h}$ must depend on the hydration level.
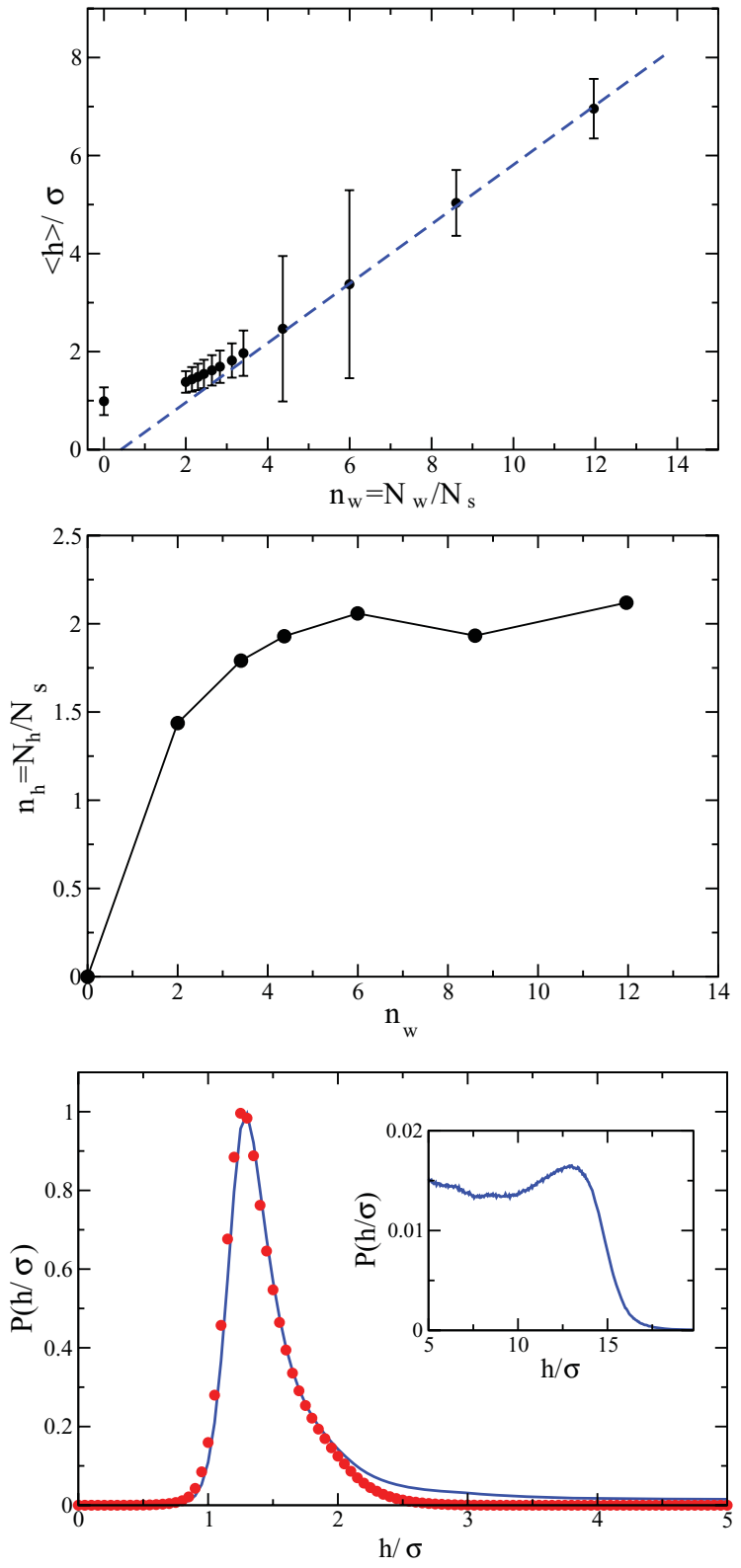

FIG. 6. (Top) Variation of the film thickness with the film water content. The dashed line represents the fitting to Eq. (6). (Middle) Estimation of the amount of "hidden" water in the Newton black film as a function of water content, estimated from Eq. (7). (Bottom) Scaled probability distribution of finding a separation, $h=\Delta \xi=\left\langle\left|\xi^{a}-\xi^{b}\right|\right\rangle$ between the intrinsic surfaces of the two monolayers. Lines and inset, results for the adhesive film at $\mathrm{n}_{w}=6$ and average surface area $33 \AA^{2}$. These results correspond to a film containing 1024 surfactants per monolayer, and the averages were computed between 148 and 160 ns. Circles, simulation results for a homogeneous film, $\mathrm{n}_{w}=2$ and average surface area $31.5 \AA^{2}$. Averages were computed between 48 and $60 \mathrm{~ns}$. The height of the main peak is proportional to the area occupied by the thin film. In the figure the height of both curves has been scaled to one.

The amount of hidden water, $\mathrm{n}_{h}$, at low water content can be estimated using the following equation:

$$
n_{h}\left(n_{w}\right)=n_{w}-A \rho_{w}\left(\left\langle h\left(n_{w}\right)\right\rangle-h_{0}\right) .
$$

The middle panel in Figure 6 represents the amount, $n_{h}$ $=N_{h} / N_{s}$, as calculated from Eq. (7). At high water content, $\mathrm{n}_{h}\left(n_{w}\right)$ converges to $2 \pm 0.1$ remaining constant around this value. This indicates that water has reached a condition 
compatible with liquid-vapor coexistence. In other words, any amount of excess water will form a liquid droplet inside the film without increasing the chemical potential. The linear increase of $\left\langle h\left(N_{w}\right)\right\rangle$ in the top panel (see Figure 6) clearly shows that the droplets condense "inside" the film, instead of forming drops outside. For smaller levels of hydration the water vapor will be undersaturated, and consequently the amount of hidden water decreases.

From the analysis above we can conclude that the film resulting from the adhesion process must have a water content close to two water molecules per surfactant. Finding the exact value of water molecules for this film requires a more involved analysis. To tackle this question we turn now to a more detailed investigation of the adhesive film.

The adhesive film consists of two "coexisting structures": one is the adhesive region, and the other one results from the formation of a water droplet containing all the excess water (see Figure 4-bottom). We have computed the probability distributions, $P\left(h ; n_{w}\right)$, of finding a particular separation, $h$, between the two intrinsic surfaces, $\xi(h)$, in a film containing $n_{w}$ water molecules per surfactant (see bottom panel in Figure 6). The probability distribution features a main peak centered at $1.3 \sigma$ followed by a slow decay and a smaller maximum at $\approx 13 \sigma$. This maximum and the slow decay are connected to the formation of the droplet in the center of the simulation box (see Figure 4), which contains the excess water. The main peak defines the thickness of the equilibrium or adhesive NBF. To quantify the properties of this film we have estimated both the average number of water molecules per surfactant and the area per surfactant for these adhesive film. This information can be extracted from the analysis of the trajectory of the big film containing 2048 surfactants. From the analysis of the thin region of the film corresponding to an area of $40 \times 30$ $\AA^{2}$ (see rectangle in Figure 4-bottom), we obtain $31.5 \pm 0.05$ $\AA^{2}$ and $\mathrm{n}_{w}=2 \pm 0.2$. Hence, the area of the adhesive film is slightly smaller than that of the initial film $\left(33 \AA^{2}\right)$. The low number of water molecules per surfactant indicates that most water molecules in this film are solvating the surfactant head groups, i.e., there is no free water present in the film.

The coexistence of these two "phases," the adhesive film and the water droplet, in the same system, is a clear indication that the adhesive NBF structure is at (constrained by fix number of surfactants) thermodynamic equilibrium, since it is formed with essentially equal characteristics over a wide range of values of hydration $\left(\right.$ see $\mathrm{n}_{w}=2,31.5 \AA^{2}$ and $\mathrm{n}_{w}=6$, $33 \AA^{2}$ in Figure 6-bottom). The adhesive region defines the saturated film, i.e., the film formed when the water vapor reaches saturation without any excess of liquid water inside the film. This film corresponds to the equilibrium NBF.

\section{The structure of the adhesive Newton black film}

In the following we investigate the structure of the adhesive film. With this purpose we have performed simulations of a system containing 512 surfactants with $\mathrm{n}_{w}=2$ water molecules per surfactant and $31.5 \AA^{2}$ per surfactant. These conditions correspond to those obtained from the analysis of the films resulting from the spontaneous adhesion process discussed above.
The radial distribution functions (RDFs) show that the sulfur atoms are surrounded at short distances by the water hydrogen atoms, which is consistent with the possibility of forming hydrogen bonds between the water hydrogens and the sulfate oxygens (see Figure 7). The S-HW RDF features a double peak, with the strong S-Na peak located at the minimum separating the two S-HW maxima. The coordination numbers, obtained from the integral up to the first minimum of the different radial distribution functions, show that each sulfate group is coordinated to $\approx 3.8$ counterions, $\approx 6.9$ water molecules, and $\approx 5.6$ sulfate groups. Regarding the coordination of the sulfate group with the water hydrogens, integration up to the first minimum of the S-HW RDF gives $\approx 2.4$ hydrogens only. This represents a small fraction, $\approx 20 \%$ of all the hydrogens associated with the water molecules that are solvating the sulfate group. Water is tightly bound to the counterions, as shown in the main peak of the Na-OW RDF (see Figure 7). We find that each $\mathrm{Na}^{+}$counterion is solvated by two water molecules. Hence the number of water molecules (that solvate the counterions) surrounding the sulfate groups is $\approx 7$. This number accounts well for the S-OW coordination number 6.9 given above, indicating there are no other water molecules solvating the surfactant head group directly. The double peak observed in the S-HW must therefore be connected to the water molecules solvating the $\mathrm{Na}^{+}$ counterions.

A more quantitative view of the adhesive film structure can be obtained by computing the intrinsic density profiles (see Figure 8). In the adhesive state water never reaches the bulk density anywhere in the film, confirming the absence of bulk water in the Newton black film. This is consistent with our estimate regarding the amount of solvation water, which is about two water molecules per surfactant. According to the analysis performed in Sec. III C (see Figure 6-middle), 1.5 water molecules per surfactant are "hidden" water, and the remaining, about 0.5 , would be contributing towards the film volume. Figure 6-bottom (circles) show that the probability distribution of finding a particular separation in the adhesive film is asymmetric, with a smooth decay for large separations
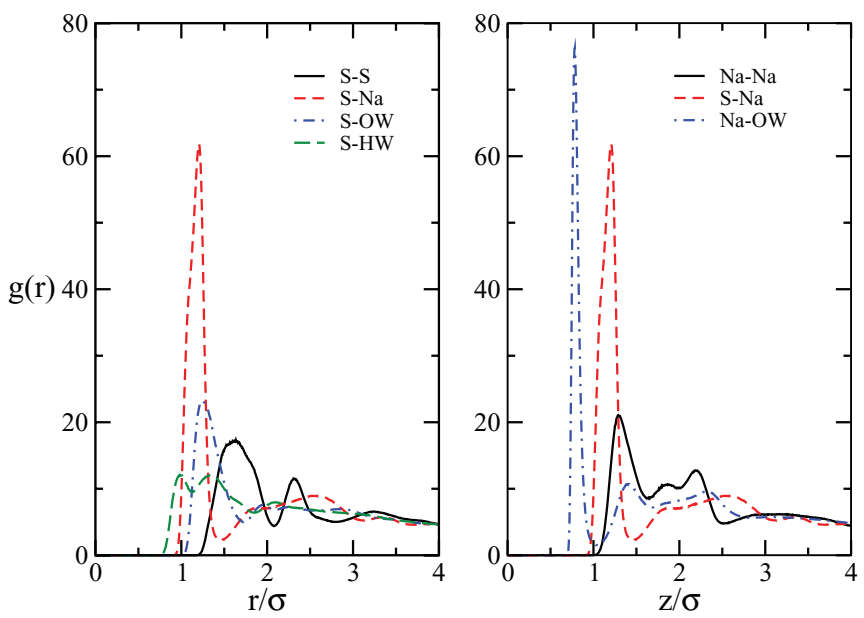

FIG. 7. Radial distribution function of sulfur-sulfur (S-S), sulfur-water oxygen (S-OW), sulfur-water hydrogen (S-HW), sulfur-sodium (S-Na), and sodium-sodium (Na-Na) pairs for the adhesive film $\left(\mathrm{n}_{w}=2,31.5 \AA^{2}\right)$. 


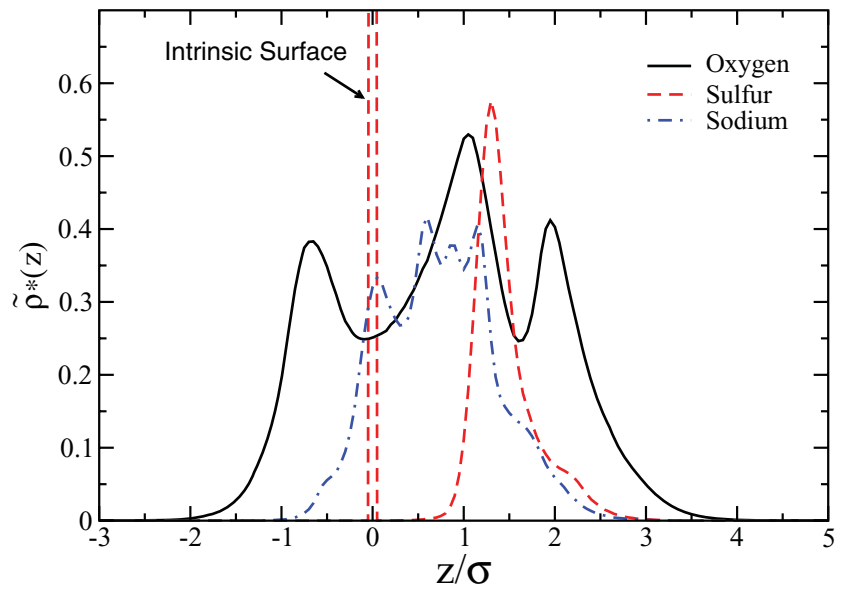

FIG. 8. Intrinsic density profiles of the adhesive film, $\mathrm{n}_{w}=2.0$ and $31.5 \AA^{2}$.

that fits well into an exponential shape, rather than the Gaussian decay that could be expected from normal undulations and breathing modes of a perfect monolayer. That suggests the water structure on the film plane must be slightly heterogeneous, with some water molecules or very small water clusters creating isolated protrusions that contribute to the smooth decay of $P(h)$. Preliminary analysis (not presented here) of the fluctuation spectrum for $h$ separated in the different transverse wave vectors support this view.

The absence of bulk water inside the film is compatible with previous experiments ${ }^{1,17}$ and simulations. ${ }^{22}$ We note, however, that the traditional image inferred from the analysis of the experiments, namely, a NBF consisting of surfactants coating the aqueous core, with water excluded from the aliphatic region, is not completely accurate. Our intrinsic profiles clearly show a significant penetration of water in the aliphatic region, with a well defined water layer around the surfactant head groups, as shown by the prominent peak in the water intrinsic profile at $z / \sigma \approx-1$. The sodium counterions distribute above, below, and in the sulfate plane. This counterion distribution is not compatible with a traditional double layer structure, where the sodium ions would be arranged in a plane below the head groups. Finally the intrinsic profiles clearly show that the two monolayers are in contact. Hence experiments measuring the disjoining pressure would find a strong repulsion connected to the direct monolayermonolayer interactions.

We have estimated the total thickness of the film from the hydrocarbon chain intrinsic profile (see Figure 9). The thickness defined as the distance between the two inflection points in the two hydrocarbon-vacuum interfaces is $2.8 \mathrm{~nm}$. This thickness is of the same order as that reported in neutron scattering and $\mathrm{X}$-ray reflectivity experiments. ${ }^{17,18}$

\section{E. The Newton black film interfacial fluctuations and bending elasticity}

The elasticity of the Newton black films can be quantified by performing an spectral analysis of the thermal fluctuations of the intrinsic surface. Once the surface is defined, and in order to obtain the capillary wave spectrum, we fit the intrinsic

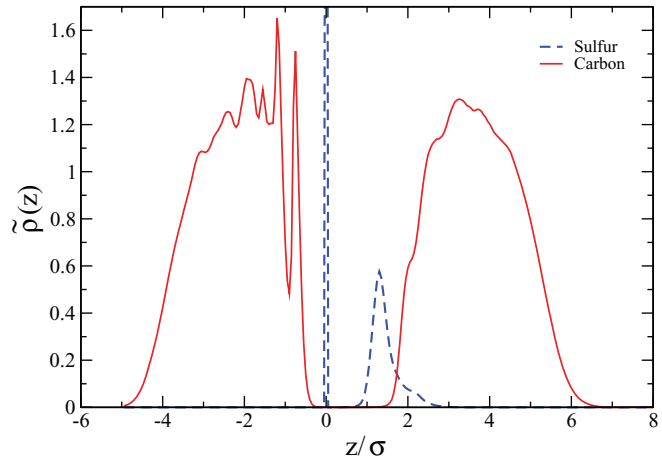

FIG. 9. Intrinsic density profile of the aliphatic chain for the adhesive of the adhesive film, $\mathrm{n}_{w}=2.0$ and $31.5 \AA^{2}$.

surface to the following Fourier series:

$$
\xi\left(\mathbf{R}, q_{u}\right)=\sum_{|\mathbf{q}| \leq q_{u}} \hat{\xi}_{q} e^{i \mathbf{q} \cdot \mathbf{R}},
$$

with the wave-vector, $\mathbf{q}_{\chi}=2 \pi n_{\chi} / L_{\chi}$, and $n_{\chi}=0, \pm 1$, $\pm 2, \cdots$, such that $\chi=x$ or $y$. The upper limit, $q_{u}=2 \pi / \lambda_{c}$, is defined by the wavelength cutoff, which has molecular dimensions $\lambda_{c}=2 \pi / \sigma, \sigma$ being a characteristic molecular size. As this fit is performed using a continuous triangulated intrinsic surface and not using a discrete number of points, the procedure does not depend significantly on the choice of $q_{u}$. Equation (8) can then be used to quantify the mean square fluctuation of the intrinsic surface, ${ }^{37}$

$$
\gamma(q)=\frac{k_{B} T}{q^{2}\left\langle\left|\hat{\xi}_{q}\right|^{2}\right\rangle A_{0}},
$$

where $\gamma(q)$ is an effective surface tension, which becomes the macroscopic surface tension, $\gamma_{0}$, in the limit $q=0$. Equation (9) furnishes a route to quantify the bending modulus, $\kappa$, of the films through ${ }^{50,51}$

$$
\frac{1}{\gamma(q) q^{2}}=\left\langle\left|\hat{\xi}_{q}\right|^{2}\right\rangle A_{0}=\frac{k_{B} T}{\gamma_{0} q^{2}+\kappa q^{4}} .
$$

Because the Newton black films investigated in this work are under tension, both the $q^{2}$ (surface tension) and the $q^{4}$ (bending) terms should contribute to the fluctuation spectrum. We note that for fluctuations involving short wavelengths, $q \sigma>2$, Eq. (9) becomes inaccurate, as the fluctuations depend on the method used to construct the intrinsic surface. ${ }^{49}$

Before we discuss the results for the bending modulus it is instructive to analyze the correlations between the two SDS monolayers. In this way we can investigate whether their fluctuations are independent from each other. The correlations can be computed from the analysis of the corresponding Fourier coefficients, $\hat{\xi}_{q}$ (see Eq. (8)). When the two layers are correlated, i.e., they move as a single entity, the correlation, $2\left\langle\hat{\xi}_{q}^{a} \hat{\xi}_{q}^{b}\right\rangle /\left(\left\langle\hat{\xi}_{q}^{a}\right\rangle^{2}+\left\langle\hat{\xi}_{q}^{b}\right\rangle^{2}\right)=1$, whereas the correlation is zero when the fluctuations are not correlated. Figure 10 shows the correlations between the two monolayers as a function of water content. For the systems with higher water content, $\mathrm{n}_{w} \approx 12$, we find that the monolayers move independently in the whole range of wavevectors investigated here. This is compatible with the results obtained above for the sulfur intrinsic profiles (see Figure 1), which shows that 


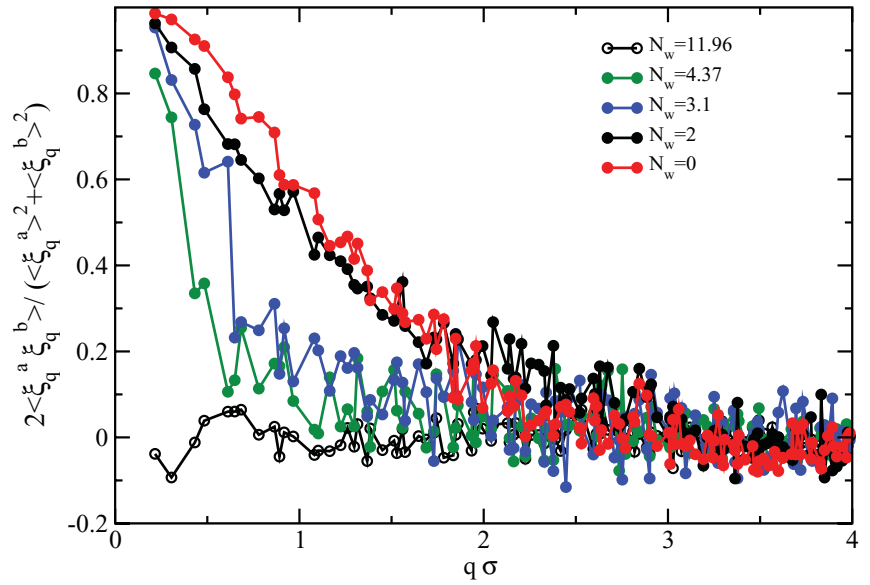

FIG. 10. Correlation between the Fourier components of the two monolayer intrinsic surfaces, denoted as "a" and "b." The data correspond to films containing 512 surfactants and area $33 \AA^{2}$.

the intrinsic density profile of one of the monolayers computed with respect to the intrinsic surface defined by the other monolayer follows a Gaussian distribution. For films featuring adhesive behavior ( $\lesssim 6$ water molecules per surfactant), we start to observe correlations at small wave-vectors, which correspond to domains of about $q^{-1} \approx 10 \AA$. The correla-
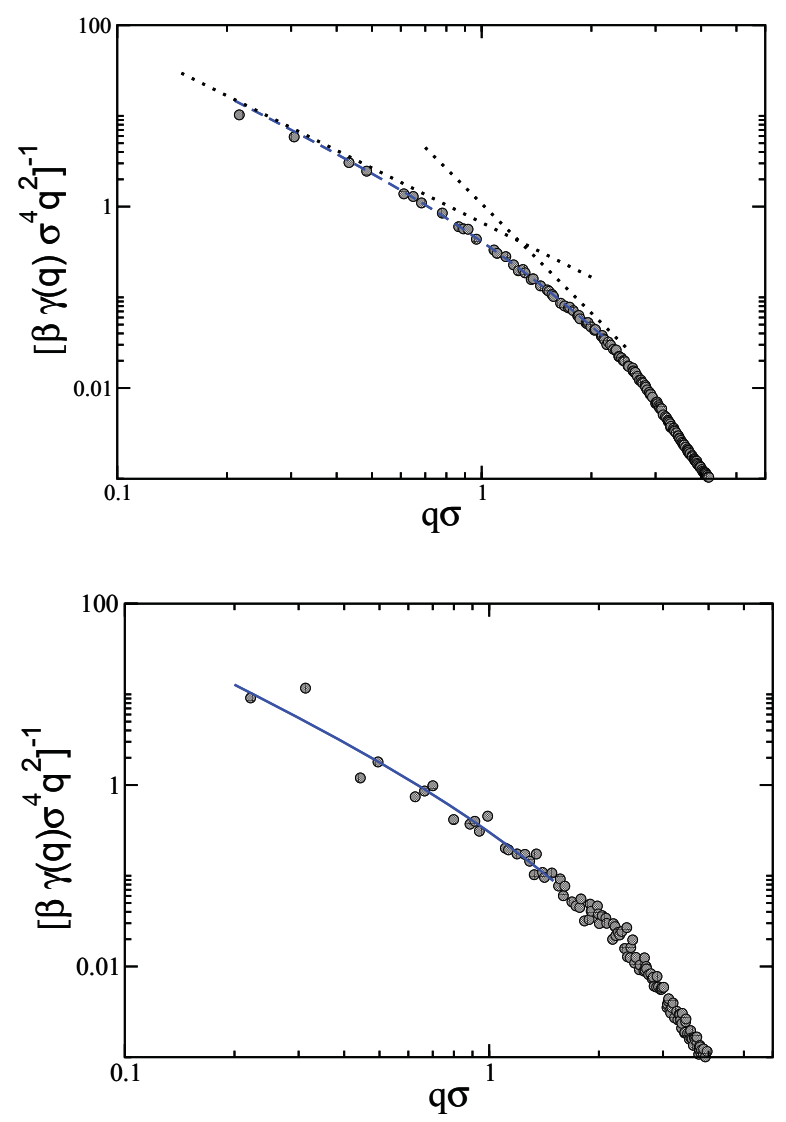

FIG. 11. Fluctuation spectrum of Newton black films as a function of water content. (Top) $\mathrm{n}_{w}=11.96,33 \AA^{2}$. Circles, simulation data; lines, fit to Eq. (10). The dotted lines show the individual contributions $q^{2}$ and $q^{4}$ in Eq. (10). (Bottom) Fluctuation spectrum of the adhesive film, $\mathrm{n}_{w}=2$, $31.5 \AA^{2}$. Circles, simulation data; full line, fit to Eq. (10) using the simulated surface tension and $\kappa$ as free parameter. See text for details. tion between the surfactant layers becomes more significant in thinner films, where long wavelength fluctuations, $q \sigma \rightarrow 0$, are completely correlated $2\left\langle\hat{\xi}_{q}^{a} \hat{\xi}_{q}^{b}\right\rangle /\left(\left\langle\hat{\xi}_{q}^{a}\right\rangle^{2}+\left\langle\hat{\xi}_{q}^{b}\right\rangle^{2}\right) \rightarrow 1$, i.e., the two layers fluctuate as a single entity (see Figure 10). This is the physical picture that defines the adhesive Newton black film $\left(\mathrm{n}_{w}=2\right)$. Otherwise, for all the systems investigated here, the fluctuations are fully uncorrelated for $q \sigma \gtrsim 3$.

In the following we report the spectral analysis of the thermal fluctuations of the SDS films. As shown in Figure 10 , for $\mathrm{n}_{w}=11.96$ the two edges of the slab exhibit uncorrelated fluctuations over the whole $q$ interval considered in this work. Hence, we have computed the fluctuations for the two monolayers independently. The results reported in Figure 11 correspond to the average of the two monolayers. We have fitted the fluctuation spectrum to Eq. (10), considering wave-vectors in the interval $0.2<q \sigma<2 .{ }^{52}$ The higher $q$ limit is necessary to avoid the inclusion of short wavelength fluctuations, which appear beyond the threshold for the protrusion modes given by $q \gtrsim 2 \pi / d$, where $d$ is the thickness of one monolayer. Considering the SDS thickness of $\sim 10 \AA$ (Ref. 28) we get a cutoff of $q \sigma \gtrsim 2$, which justifies the interval chosen above. In films with high water content, $\mathrm{n}_{w}=11.96$, the fitting of our data to Eq. (10) is excellent. The results for the SDS monolayer surface tension and the bending modulus are $\beta \gamma_{0} \sigma^{2}=1.5 \pm 0.3$ and $\beta \kappa=0.93 \pm 0.2$, respectively. The surface tension obtained from this approach is in very good agreement with the surface tension of the monolayer computed through the microscopic pressure tensor route, $1.46 \pm 0.05$. Note that these values would describe the macroscopic limit of a single SDS monolayer on a water surface, and they do not include any coupling effect between the two edges of the water slab. For lower water content $\mathrm{n}_{w}=8.6$ (not shown), we find that the fluctuations of the two edges are still uncoupled, and the estimation for the surface tension is $\beta \gamma_{0} \sigma^{2}=1.3 \pm 0.3$ vs. $1.4 \pm 0.1$ obtained from the pressure tensor route, and the bending modulus is $\beta \kappa=1.1 \pm 0.2$. All these data are within the error bars given above for the thicker slab $\left(\mathrm{n}_{w}=11.96\right)$.

In the adhesive film, $\mathrm{n}_{w}=2$ and area $31.5 \AA^{2}$, both monolayers are strongly correlated and they move as a single entity for $q \sigma \lesssim 1$ (see Figure 10). Because $\left\langle\left|\hat{\xi}_{q}^{a}\right|^{2}\right\rangle=\left\langle\left|\hat{\xi}_{q}^{b}\right|^{2}\right\rangle$ we can compute the fluctuations considering any of the monolayers. The adhesive film exhibits very slow dynamics, this may explain why the fluctuation spectrum becomes noticeably noisier at low $q$ values. Our results suggest that significantly longer simulation times than those considered here, $50 \mathrm{~ns}$, are required to improve the statistics at low $q$ values. Using our data and the surface tension $\beta \gamma \sigma^{2}=1.9$ \pm 0.05 obtained through the pressure tensor route, our best estimate for the bending modulus from equation (10) is $\beta \kappa=1.4 \pm 0.3$. Notice that these values for the adhesive film $\mathrm{n}_{w}=2$ describe the macroscopic behavior of the film as a whole, unlike the values of $\gamma$ and $\kappa$ obtained with $\mathrm{n}_{w}=11.96$ and 8.6, which correspond to a single SDS monolayer.

\section{CONCLUSIONS AND FINAL REMARKS}

We have investigated the properties of sodium dodecyl sulfate Newton black films (SDS-NBFs) at $\mathrm{T}=298 \mathrm{~K}$, as a 
function of water content and at a fixed area per surfactant of $33 \AA^{2}$, which is near the one reported in experimental studies of SDS-NBFs. The intrinsic density and orientational profiles of water were computed using an approach that removes the averaging effect of the thermal capillary waves. This technique relies on the definition of an intrinsic surface. Once this is defined it is possible to construct intrinsic profiles as well as to quantify the film fluctuation spectrum.

The intrinsic profiles clearly show the existence of a well defined water solvation layer that solvates the surfactant head group both in the aliphatic and aqueous regions. This result differs from the image inferred from models found in the literature, where the water molecules are located inside the film core only. Our results indicate that the solvation layer comprises about two water molecules per surfactant. This solvation structure is preserved in a wide range of film thicknesses, from an infinite thickness (surfactant monolayers) down to monolayer-monolayer separations of the order of $10 \AA$. For thinner films the water structure in the film core is strongly modified, whereas it remains unaltered in the aliphatic region, even for very low hydration levels, $\lesssim 2$ water molecules per surfactant. Near complete dehydration, $\mathrm{n}_{w} \approx 1.5$ the surfactant monolayers are in direct contact and the few water molecules remaining in the film are expelled to the aliphatic region, forming a double layer structure.

The intrinsic profiles provide a new view on how the water structure is perturbed inside the film. The intrinsic density profile features a characteristic oscillatory behavior reaching the bulk density in about $10 \AA$ form the monolayer plane. This result defines a clear length scale for water correlations, which is missed in previous analysis based on mean profiles.

We have quantified the correlations between the film monolayers. The monolayer fluctuations in films with high water content, $\mathrm{n}_{w} \gtrsim 8$, are uncorrelated for the whole range of wave-vectors investigated in this work $\left(q \gtrsim 0.6 \AA^{-1}\right)$, i.e., the monolayers move independently from each other. The fluctuation spectrum can be accurately modeled in terms of the surface tension and the bending modulus as described by Helfrich equation. The bending modulus of the film monolayers, $\kappa \approx k_{B} T$, agrees with previous computations of surfactant monolayers. The fluctuation spectrum of thinner films $(\approx 2$ water molecules per surfactant) is noisier at low wave vectors $q$. This makes difficult a direct quantification of the bending modulus through Helfrich equation. It may be possible that this behavior is connected to the formation of a glass. Further work is required to fully characterize this point.

We have shown the existence of adhesive behavior in the SDS black films. This is the most important result of this work. Although adhesion can be inferred from experiments of SDS stabilized emulsions, our work is to the best of our knowledge, the first computer simulation study reporting such behavior. Investigations of films of different sizes show that the adhesion behavior is reproducible. This result strongly suggests that the adhesive film corresponds to an equilibrium state for the slab geometry investigated here. We want to point out that the precise thickness of the equilibrium state will depend on the experimental conditions, the surfactant surface concentration being an important variable in this instance. For the system size and surfactant concentrations investigated in this work, we find that films with less than six water molecules per surfactant become unstable, and evolve over time to a structure containing the adhesive film and one water droplet. Large scale simulations of 2048 surfactants show that this process takes about $100 \mathrm{~ns}$ at $298 \mathrm{~K}$, and can therefore be investigated in atomistic simulations within reasonable computational times. From the analysis of the simulations we find that the adhesive or equilibrium state of the Newton black film contains two water molecules per surfactant, the area per surfactant being $31.5 \AA^{2}$, and the monolayers are essentially in contact. The overall film thickness is in good agreement with that inferred from neutron scattering experiments of oil-SDSwater emulsions and x-ray reflectivity experiments of SDSwater black films.

Finally, we would like to note that it is possible to rationalize the adhesive behavior using a thermodynamic model that describes the adhesion process as a transition to a partial wet state. A theoretical proof of this notion will be reported in a forthcoming publication.

\section{ACKNOWLEDGMENTS}

We would like to acknowledge the Imperial College High Performance Computing Service for providing computational resources. Financial support for this work was provided by The Royal Society and the Dirección General de Investigación, Ministerio de Ciencia y Tecnología of Spain, under Grant No. FIS2010-22047-C05, and by the Comunidad Autónoma de Madrid under the R\&D Program of activities MODELICO-CM/S2009ESP-1691. H.M. would like to thank the Universidad Autonoma de Madrid for the award of a FPUUAM doctoral grant.

${ }^{1}$ V. J. Bergeron, J. Phys.: Condens. Matter 11, R215 (1999).

${ }^{2}$ D. Exerowa, R. Cohen, and A. Nikolova, Colloids Surf. 24, 43 (1987).

${ }^{3}$ V. J. Bergeron, A. Waltermo, and P. M. Claesson, Langmuir 12, 1336 (1996).

${ }^{4}$ J. J. Benattar and M. Nedyalkov, Angew. Chem., Int. Ed. 45, 4186 (2006).

${ }^{5}$ M. Nedyalkov, V. Petkova, and D. Platikanov, Colloids Surf., A 220, 35 (2003)

${ }^{6}$ M. N. Jones, K. J. Mysels, and P. C. Sholten, Trans. Faraday Soc. 62, 1336 (1966).

${ }^{7}$ A. Waltermo, P. M. Claesson, S. Simonson, E. Manev, I. Johansson, and V. J. Bergeron, Langmuir 12, 5271 (1996).

${ }^{8} \mathrm{~J}$. Israelachvili, Intermolecular and Surface Forces, 1st ed. (Academic, New York, 1991).

${ }^{9}$ D. Exerowa, T. Kolarov, and K. H. Khristov, Colloids Surf. 22, 171 (1987).

${ }^{10}$ J. Faraudo and F. Bresme, Phys. Rev. Lett. 92, 236102 (2004).

${ }^{11}$ J. Faraudo and F. Bresme, Phys. Rev. Lett. 94, 077802 (2005).

${ }^{12}$ V. J. Bergeron and C. J. Radke, Langmuir 8, 3020 (1992).

${ }^{13}$ S. Leikin, V. A. Parsegian, D. C. Rau, and R. P. Rand, Annu. Rev. Phys. Chem. 44, 369 (1993).

${ }^{14}$ S. Marcelja and N. Radic, Chem. Phys. Lett. 42, 129 (1976).

${ }^{15}$ N. A. M. Besseling, Langmuir 13, 2113 (1997).

${ }^{16}$ J. Israelachvili and H. Wennerström, Nature (London) 379, 219 (1996).

${ }^{17}$ O. Bélorgey and J. J. Benattar, Phys. Rev. Lett. 66, 313 (1991).

${ }^{18}$ P. Poulin, F. Nallet, B. Cabane, and J. Bibette, Phys. Rev. Lett. 77, 3248 (1996).

${ }^{19}$ J. Bibette, F. L. Calderon, and P. Poulin, Rep. Prog. Phys. 62, 969 (1999).

${ }^{20}$ P. Poulin and J. Bibette, Phys. Rev. Lett. 79, 3290 (1997).

${ }^{21}$ Z. Gamba, J. Hautman, J. C. Shelley, and M. L. Klein, Langmuir 8, 3155 (1992).

${ }^{22}$ F. Bresme and J. Faraudo, Langmuir 12, 5127 (2004).

${ }^{23}$ F. Bresme and J. Faraudo, Mol. Simul. 32, 1103 (2006). 
${ }^{24}$ W. H. Yang, R. L. Wu, V. Kong, X. Zhang, and X. Yang, J. Phys. Chem. B 113, 8332 (2009).

${ }^{25}$ S. di Napoli and Z. Gamba, J. Chem. Phys. 132, 075101 (2010).

${ }^{26}$ W. Yang and X. Yang, J. Phys. Chem. B 114, 10066 (2010).

${ }^{27}$ A. Kabalnov and H. Wennerström, Langmuir 12, 276 (1996).

${ }^{28}$ H. Martínez, E. Chacón, P. Tarazona, and F. Bresme, "The intrinsic interfacial structure of ionic surfactant monolayers at water-oil and water-vapour interfaces," Proc. R. Soc. London, Ser. A (in press).

${ }^{29}$ M. G. Martin and J. I. Siepmann, J. Phys. Chem. B 102, 2569 (1998).

${ }^{30}$ W. D. Cornell, P. Cieplak, C. I. Bayly, I. R. Gould, K. M. Merz, D. M. Ferguson, D. C. Spellmeyer, T. Fox, J. W. Caldwell, and P. A. Kollman, J. Am. Chem. Soc. 117, 5179 (1995).

${ }^{31}$ J. C. Shelley, K. Watanabe, and M. L. Klein, Int. J. Quantum Chem. 17, 103 (1990).

${ }^{32}$ L. X. Dang, J. Am. Chem. Soc. 117, 6954 (1995).

${ }^{33}$ J. L.F. Abascal and C. Vega, J. Chem. Phys. 123, 234505 (2005).

${ }^{34}$ F. Bresme, E. Chacón, and P. Tarazona, Mol. Phys. 108, 1887 (2010).

${ }^{35}$ T. Darden, D. York, and L. Pedersen, J. Chem. Phys. 98, 10089 (1993).

${ }^{36}$ B. Hess, J. Chem. Theory Comput. 4, 435 (2008).

${ }^{37}$ E. Chacón and P. Tarazona, Phys. Rev. Lett. 91, 166103 (2003).

${ }^{38}$ D. Lacasse, G. S. Grest, and A. J. Levine, Phys. Rev. Lett. 80, 309 (1998).

${ }^{39}$ S. Senapati and M. L. Berkowitz, Phys. Rev. Lett. 87, 176101 (2004).

${ }^{40}$ M. González-Melchor, F. Bresme, and J. Alejandre, J. Chem. Phys. 122, 104710 (2005)

${ }^{41}$ R. Evans, Adv. Phys. 28, 143 (1979).
${ }^{42}$ S. A. Pandit, D. Bostick, and M. L. Berkowitz, J. Chem. Phys. 119, 2199 (2003).

${ }^{43}$ P. Tarazona and E. Chacón, Phys. Rev. B 70, 235407 (2004).

${ }^{44}$ J. Chowdhary and B. M. Ladanyi, J. Phys. Chem. B 110, 15442 (2006).

${ }^{45}$ F. Bresme, E. Chacón, P. Tarazona, and K. Tay, Phys. Rev. Lett. 101, 056102 (2008).

${ }^{46}$ F. Bresme, E. Chacón, and P. Tarazona, Phys. Chem. Chem. Phys. 10, 4704 (2008).

${ }^{47}$ L. B. Partay, G. Hantal, P. Jedlovszky, A. Vincze, and G. Horvai, J. Comput. Chem. 29, 945 (2008).

${ }^{48}$ M. Jorge, P. Jedlovszky, and N. D. S. Cordeiro, J. Phys. Chem. C 114, 11169 (2010).

${ }^{49}$ E. Chacón, M. Fernández, D. Duque, R. Delgado-Buscalioni, and P. Tarazona, Phys. Rev. B 80, 195403 (2009).

${ }^{50}$ W. Helfrich, Z Naturforsch. C 28, 693 (1973).

${ }^{51}$ S. A. Safran, Statistical Thermodynamics of Surfaces, Interfaces and Membranes (Addison-Wesley, Reading, MA, 1994).

${ }^{52}$ We note that the correlations and the qualitative behavior of $\gamma(q)$ with $q$ are independent of the approach used to define the intrinsic surface. The quantitative predictions may in principle depend on this definition though. For the system investigated in this work, we found that the intrinsic surfaces obtained using the Delaunay approach employed in this paper or the intrinsic sampling method produce the same bending modulus within statistical error.

${ }^{53}$ K. J. Schweighofer, U. Essmann, and M. L. Berkowitz, J. Phys. Chem. B 101, 3793 (1997). 